\title{
Estratégias de produção e organização de informações na web: conceitos para a análise de documentos na internet
}

\author{
Carlos d'Andréa \\ Mestre em ciência da informação e especialista em gestão estratégica \\ da informação pela ECI/UFMG. \\ E-mail: carlosdand@gmail.com
}

\begin{abstract}
Resumo
Este artigo visa a apresentar e articular conceitos importantes para a compreensão, sob a ótica da ciência da informação, das especificidades e potencialidades do documento eletrônico no ambiente hipertextual da web. Para isso, são resgatados termos como "documento", "cadeia documental" e "unidade de informação", que são aproximados a conceitos característicos do ambiente digital como "hipertextualidade" e "arquitetura da informação". Este trabalho é parte dos estudos realizados na dissertação "Estratégias de produção e organização de informações na web: uma análise de sites turísticos", apresentada em abril de 2005 ao Programa de Pós-graduação em Ciência da Informação da Universidade Federal de Minas Gerais (UFMG), que teve como objetivo final (não contemplado neste artigo) a proposição de uma metodologia de análise de sites que privilegie a identificação e compreensão de seus processos de produção e organização de informações.
\end{abstract}

\section{Palavras-chave}

Arquitetura da informação. Avaliação de sites. Documento eletrônico. Internet. Organização da informação.

\section{Strategies of production and organization of information in web: analysis of documents in Internet}

\begin{abstract}
This paper presents the concepts that are important for the understanding, under the light of Information Science, of the specificities and potentiality of the electronic document in the hypertextual environment of the WWW. To achieve this, terms such as document, documentation chain and information unit are brought together to concepts that characterize the digital environment such as hypertextuality and information architecture. The ideas presented here are part of the studies developed for the dissertation "Strategies for the creation and organization of information in the WWW: an analysis of touristic sites", presented in April of 2005 at the Graduate Program in Information Science of the School of Information Science of the Federal University of Minas Gerais, main objective was to present a methodology for analysis of sites (not included in this paper) that takes into account the identification and understanding of the processes of creation and organization of information.
\end{abstract}

\section{Keywords}

Internet. Eletronic document. Information organization. Information architecture. Assessment of sites.
A emergência da chamada sociedade da informação acelerou um processo de mudanças socioculturais caracterizado, entre outros fatores, pela rápida disseminação de informações e facilidade de comunicação em nível global, principalmente por meio das chamadas tecnologias da informação e comunicação (TICs). Com o advento dessas ferramentas, a publicação e o acesso a informações de diferentes naturezas passam a ocorrer em escala nunca antes vista. Diferentes atores sociais ou instituições podem assumir, ainda que informalmente, a função de uma unidade de informação e conseguir a atenção do leitor-usuário durante a busca por informações na web.

Miranda (2000) destaca a "incontrolabilidade dos conteúdos" produzidos e disponibilizados nos meios eletrônicos como uma marca da sociedade contemporânea. Essa facilidade de contato e expressão reforça ainda mais a pluralidade de vozes que podem se pronunciar sobre uma região, trazendo mais e novos traços de identificação para as redes. Conforme afirma Barreto (1998, p.126),

a comunicação eletrônica veio definitivamente libertar o texto e a informação de uma ideologia envelhecida e autoritária dos gestores da recuperação da informação, defensores de uma pretensa qualidade ameaçada, os fatais intermediários e porta-vozes que vêem seus poderes ameaçados cada vez mais pela facilidade da convivência direta entre os geradores e consumidores da informação.

Tamanha diversidade tanto possibilita potencial multiplicidade de vozes manifestando-se sobre o mesmo tema, quanto faz com que seja ainda mais importante lançar um olhar criterioso sobre o universo da web, especialmente visando a caracterizar e analisar os sites.

Uma avaliação eficiente de informações disponíveis na internet depende ainda da consolidação de uma área de estudos sobre o tema. Mesmo considerando que o ato de avaliar algo é, por natureza, uma ação subjetiva, isto é, depende da interpretação e ponto de vista de um sujeito específico, acreditamos ser relevante e possível o desenvolvimento de reflexões, especialmente no ambiente 
diverso e plural da internet. Smith (1997) ressalta o papel histórico do bibliotecário no processo de seleção das informações, lembrando que ele pode estender para o ambiente on-line o tradicional trabalho de avaliar, selecionar e organizar a informação publicada, em uma posição reforçada por Tillman (2003) e Tomael et al. (2001), entre outros, reforçando a identificação dos processos avaliativos com o campo de estudos da ciência da informação.

Trabalhos de referência como os de Tillman (2003), Alexandre e Tate (1996) e Smith (1997) em comum têm o desenvolvimento de listas de critérios de avaliação dos recursos oferecidos por um web site. Trata-se de grandes check lists a serem aplicados pelo avaliador de um site, visando a identificar e julgar o maior número possível de elementos disponíveis na página. As listas de itens a serem avaliados produzidos pelos autores mais citados foram o ponto de partida para algumas pesquisas realizadas no Programa de Pós-Graduação em Ciência da Informação (PPGCI) da Escola de Ciência da Informação da UFMG, principalmente as de Nascimento (2000), Carvalho (2001) e Vilella (2003), que procuraram adaptar listas de critérios de avaliação de sites a diferentes universos e temas, gerando assim desdobramentos nessa área de pesquisas e contribuindo para uma evolução das pesquisas.

A perspectiva apresentada no presente trabalho, no entanto, em grande parte difere das abordagens citadas anteriormente. Com vistas ao desenvolvimento de um olhar mais qualitativo, menos preso à simples conferência de todos os detalhes que compõem um site, propomos aqui um método que privilegia aspectos mais estruturais e processuais adotados pelas equipes da unidade de informação ao produzir e organizar determinado site.

Considerando que a "ciência da informação está ligada ao corpo de conhecimentos relativos à origem, coleta, organização, estocagem, recuperação, interpretação, transmissão, transformação e uso da informação" (Borko, 1968), procuramos resgatar da área conceitos tradicionalmente usados para caracterizar os atores e objetos envolvidos nos processos ligados à informação, tais como "documento", "unidade de informação", "organização da informação" e "cadeia documental", para identificar que mudanças podem ser identificadas na "migração" do espaço da informação para o ambiente digital e hipermidiático.

Ao considerarmos as especificidades do documento eletrônico, identificamos e discutimos como acontece o processo de produção e organização de suas informações, uma vez que os procedimentos tradicionalmente adotados em bibliotecas, por exemplo, não parecem ser perfeitamente adequados a estes objetos. Do mesmo modo, julgamos importante identificar a relação entre as unidades de informação responsáveis pelos sites e as informações neles disponibilizadas, sempre com o objetivo de compreender sua influência na seleção e organização das informações veiculadas. Consideramos ainda que um site deve ser analisado também quanto à adequação das informações ao ambiente hipertextual potencialmente oferecido pela web.

Assim, procuramos, neste artigo, levantar e articular conceitos relavantes para uma análise de site, considerando, em suma, os processos de produção/coleta e organização das informações disponíveis nos sites, as características do documento eletrônico, a especificidade do ambiente hipertextual da web e a natureza da unidade de informação responsável pelo site. Em d'Andréa (2005), estes conceitos foram a base para a elaboração de um método de análise de sites, que considerou ainda a especificidade do conteúdo do site. $\bigcirc$ método de análise não será apresentado neste artigo.

\section{O CONCEITO DE DOCUMENTO E A WEB}

Como a biblioteconomia é uma das áreas fundadoras da ciência da informação, devemos considerar os processos de organização da informação como um dos campos principais de atuação desta área de conhecimento. Considerando que os

objetivos das atividades documentais são selecionar, na massa de informações veiculadas, os elementos de conhecimento, fornecendo a qualquer pessoa as informações de que ela necessita, no momento que as solicita, e ainda conservar estas informações atualizadas, sem alterá-las (GUINCHAT; MENOU, 1994, p. 19),

podemos afirmar que, nas diferentes épocas históricas, e de acordo com as condições materiais e de conhecimento disponíveis, as instituições especializadas adotaram diferentes técnicas para, em última instância, facilitar o acesso das pessoas às informações organizadas. Os desafios de organização da informação são, no entanto, muito anteriores ao desenvolvimento das tecnologias atuais, tornando extremamente relevante um resgate dos conceitos tradicionalmente usados no contexto das unidades de informação, como as bibliotecas, visando a identificar mudanças e possíveis deficiências e melhorias nos processos hoje adotados.

Nessa linha, considerando que a informação é a matériaprima de estudo e trabalho para a área, é fundamental 
conhecermos o suporte em que ela se apresenta, o chamado "documento". Para Guinchat e Menou (1994, p.41), documento "é um objeto que fornece um dado ou uma informação". Svenonius (2000, p.8) destaca que, na literatura de organização da informação, há uma aproximação do sentido de documento com o conceito de suporte. "Informação é uma abstração, mas os documentos que a contêm estão expressos em algum meio", como papel, pedra ou chips de computador.

Este conceito de documento, no entanto, está baseado em um contexto de trabalho pouco influenciado por artefatos tecnológicos. Devemos considerar que as mudanças estruturais no processo informacional causadas pela digitalização dos documentos e pela comunicação eletrônica trouxeram impactos definitivos na configuração dos documentos, inclusive em sua estrutura interna e lógica de funcionamento. Como afirma Pédauque (2003), "a forma eletrônica está revolucionando o conceito de documento".

O autor propõe debatermos este novo documento a partir de três aspectos complementares: pela forma, como signo e como meio, que resultam em duas grandes diferenças do documento eletrônico em relação ao tradicional. No aspecto forma, é identificada uma vinculação total entre o conteúdo e a estrutura em que ele se apresenta, sendo redutor analisar o conteúdo sem considerar conjuntamente seu suporte, uma vez que a disposição das informações (em um site, por exemplo) explora os recursos oferecidos pela estrutura em que elas se apresentam e depende desta para serem acessadas plenamente pelo usuário. Trata-se, enfim, de intensa simbiose entre forma e conteúdo, que tradicionalmente são aspectos analisados de forma isolada.

Nos aspectos signo e meio, o documento eletrônico exige maior participação do usuário/leitor na construção do significado, uma vez que a própria configuração do documento exige alguma interação, que depende do conhecimento sobre o meio e o tema e de procedimentos técnicos. No caso da web, a hipertextualidade é a característica que marca esta intervenção "obrigatória" do usuário na reconstrução do documento - um site será plenamente explorado somente durante o processo de navegação pelo usuário, que se torna responsável ainda pelo processo de legitimação do documento no enorme universo de informações da internet.

\section{CADEIA DOCUMENTAL}

Considerando que o gerenciamento dos documentos é uma atividade extremamente complexa e que exige a atuação de diferentes áreas do conhecimento, Guinchat e Menou (1994, p.30) estruturam o processo de atividades e operações dentro de uma "cadeia documental", na qual são identificadas cinco etapas encadeadas: coleta de documentos; controle e registro material do documento; armazenamento; pesquisa; difusão da informação.

$\mathrm{Na}$ coleta de documentos, acontecem a localização dos documentos, a seleção e os procedimentos de aquisição, que "exigem um esforço constante da unidade de informação" (GUINCHAT; MENOU, 1994, p.85), uma vez que os critérios técnicos de seleção dos documentos estão diretamente ligados às características e políticas da unidade. É importante identificarmos que, no caso dos ambientes digitais e em especial da internet, esta etapa contempla não apenas a coleta de documentos produzidos externamente à unidade de informação, mas muitas vezes a produção de documentos diretamente pela UI, considerando a facilidade técnica de publicação de informações na web.

Durante o controle e registro material do documento, acontece o tratamento intelectual do documento que já pertence à unidade de informação, seguindo as etapas da descrição bibliográfica (catalogação de suas características formais, como autor, título, fonte, formato) e a descrição do conteúdo (tradução das informações para uma linguagem documental, de acordo com os interesses da unidade e de seus usuários, permitindo a classificação, indexação, resumo ou extração de dados).

A terceira etapa é o armazenamento ou arquivamento, que pode basear-se em dois grandes tipos de arranjo dos documentos: numérico (organizados por ordem de chegada) e sistemático (classificados por conteúdos). A quarta e a quinta etapas da cadeia documental são a pesquisa (a partir da memória) e difusão da informação. Elas não serão discutidas neste trabalho porque nossa opção foi por estudar os processos de coleta/produção e organização da informação, que se encerram no armazenamento.

\section{HIPERTEXTUALIDADE, ARQUITETURA DA INFORMAÇÃO E UMA NOVA CADEIA DOCUMENTAL}

Se identificamos anteriormente uma necessidade de mudanças no próprio conceito de documento a partir do desenvolvimento dos ambientes digitais de armazenamento e publicação, parece-nos inevitável que haja também alterações na cadeia documental descrita por Guinchat e Menou (1994) e adotada por unidades tradicionais de informação. 
A propagada natureza flexível e descentralizada que caracteriza a web em grande parte apóia-se na lógica de articulação de informações por meio do hipertexto, caracterizado por Le Coadic (1996, p.59) como

o veículo informático de uma informação não-linear, resultado do desmantelamento, pelo computador, da organização estritamente seqüencial do suporte de papel.

Para o autor, no papel "as estruturas físicas e lógicas estão muito próximas”, enquanto, no ambiente eletrônico ou mesmo em documentos de papel (como enciclopédias), a estrutura física é composta por "seqüências lineares de unidades independentes", que são interligadas de forma lógica e múltipla, como uma rede flexível e potencialmente aberta.

Ao considerar o objetivo principal de articular conceitos, devemos levantar e debater como o ambiente hipertextual da web altera a cadeia documental descrita anteriormente e permite que o documento eletrônico seja trabalhado explorando suas características diferenciadoras. Para Parente (1999, p.81),

"o hipertexto digital incorpora às velhas ferramentas de busca da informação uma série de outras: a lista, o repertório, o anuário, a classificação, a bibliografia, o catálogo, o index,

isto é, o ambiente hipertextual retoma recursos e procedimentos tradicionais de organização da informação, no entanto exigindo novas estratégias e rotinas de organização da informação.

A esses procedimentos atribui-se o nome de "arquitetura da informação", definida por Rosenfeld e Morville, em citação de Tristão (2002), como uma "combinação entre esquemas de organização, nomeação e navegação dentro de um sistema de informação". Para Rosenfeld e Morville (1998, p.11), o arquiteto da informação é responsável pela definição "do que o site realmente será e como ele funcionará”. Entre suas funções, está a de deixar clara a missão e a visão do site, determinar que conteúdo e funcionalidades do site vai conter, especificar como o usuário encontrará as informações (ao definir a organização, navegação, classificação e busca) e mapear como o site pode crescer e mudar futuramente.

processo de execução da arquitetura da informação exige o cumprimento de algumas etapas de trabalho, dentre as quais, a partir do trabalho de Rosenfeld e
Morville (1998), destacamos a organização da informação o e desenvolvimento de sistema de navegação.

Os esquemas de organização são lógicas de agrupamento das informações que visam a ressaltar características comuns dos itens de conteúdo. Durante o processo, podese optar pela adoção de esquemas exatos (ordem alfabética, cronológica ou geográfica, por exemplo) ou ambíguos (informação organizada em categorias definidas pelo gestor). Paralelamente à definição e implementação de um esquema de organização do site, o "arquiteto da informação" deve trabalhar a estrutura de organização, por meio da qual serão definidos os caminhos principais de navegação oferecidos aos usuários. A relação entre os itens do conteúdo e os grupos aos quais eles pertencem pode ser hierárquica, hipertextual ou modelo de banco de dados relacional.

É necessária também a adoção de uma navegação eficiente pelas informações, evitando que os usuários fiquem perdidos ao usar o site, uma vez que os processos de organização atuam de modo mais estrutural e normalmente não são percebidos pelo usuário leigo. Os diferentes tipos de sistemas de navegação são a hierárquica, global e ad hoc. A navegação hierárquica é um sistema primário de navegação extremamente importante, mas deve ser combinada com outros sistemas de navegação. A navegação global possibilita navegações complementares pela estrutura do site, como movimentos laterais e verticais que rompem com a navegação única proposta pelo sistema hierárquico, servindo, ao indicar em que seção o usuário está, para marcar o contexto para o usuário . Trata-se das opções encontradas em todas as páginas do site. A navegação local é recomendada como complemento aos sistemas de navegação global para sites mais complexos e facilita a navegação por áreas específicas de um site. Seus itens mudam de acordo com a página visitada. A navegação ad hoc não está presa aos sistemas listados anteriormente, podendo-se optar por criar links ao longo do texto associando informações que têm algum tipo de relação entre si.

Podemos identificar que parte dos procedimentos apresentada anteriormente pode ser considerada uma adaptação, uma nova versão da "cadeia documental" à qual uma unidade de informação tem de submeter-se ao trabalhar com seus documentos, sendo, portanto, conceitos relevantes na análise das estratégias de produção e organização de um site. 


\section{UNIDADES DE INFORMAÇÃO}

Devemos considerar que os processos da cadeia documental aos quais o documento é submetido estão sempre vinculados a uma estrutura institucional, que avaliará desde sua relevância até o modo como ele será disponibilizado ao usuário. À exceção de sua característica material (papel, por exemplo), um documento não possui características intrínsecas, dadas por natureza e independentes do contexto em que é analisado. É este contexto que influencia qual o peso e significado será atribuído a uma informação contida em um documento.

A unidade de informação é a instituição responsável por gerenciar um ou mais dos processos da cadeia documental, sendo composta por profissionais especializados que atuam de acordo com a sua especificidade. Para Guinchat e Menou (1994), "a proliferação de termos que designam as diversas unidades de informação traduz a enorme riqueza da informação documental", citando as bibliotecas, os arquivos, as bibliotecas especializadas, os centros ou serviços de documentação, os centros ou serviços de análise da informação, entre outros.

Com o aumento do número de usuários e com o surgimento de novas técnicas de gerenciamento da informação, nota-se hoje uma multiplicação de atividades de organismos especializados em diferentes etapas da cadeia documental. De acordo com os autores, essas unidades podem ser classificadas em três ramos de atividades: conservação e o fornecimento de documentos primários (arquivos, bibliotecas, mediatecas e museus), centros e serviços de documentação (centrados na descrição de conteúdo dos documentos) e centros e serviços de informação (especializados na resposta a questões e na exploração de informações específicas a partir de documentos primários e secundários ou coleções de dados).

O avanço da tecnologia nas últimas décadas também trouxe significativo impacto para as unidades de informação. A facilidade de produção, publicação e difusão de informações, que resultou também na proliferação de unidades "informais" de informação, assim como a possibilidade de acesso remoto a bases de dados e acervos, alterou significativamente as características das unidades tradicionais, os processos por elas executados e o perfil dos profissionais envolvidos.

Maior facilidade no acesso à informação e novas possibilidades de produção e difusão das informações exigiram o que Guinchat e Menou (1994, p.27) identificam como uma transformação das estruturas tradicionais centralizadas, fechadas e opacas, em redes de informação transparentes, fluidas e abertas, com múltiplos pontos de acesso.

Para Barreto (1997),

os fatores tecnológicos determinados por uma tecnologia intensa em inovação e velozmente mutante são os outros parceiros responsáveis pelo reposicionamento dos agentes do setor de informação, que atinge o produtor de estoques de informação, o documento de informação, a transferência da informação e sobretudo a relação do usuário com todos os demais.

Esta mudança fica ainda mais evidente em um ambiente como o da internet, no qual o usuário tem acesso às informações em tempo real (mudança na relação usuáriotempo-informação) e de forma direta (relação usuárioespaço-informação), exigindo que a atuação dos profissionais da informação concentre-se primordialmente no momento anterior ao acesso, quando cabe a ele exercer com critério as funções de coleta/produção e organização da informação.

As condições e objetivos de uma unidade de informação são variáveis fundamentais no funcionamento final da mesma, uma vez que seus "filtros" institucionais, políticos, econômicos etc. influenciam em toda a cadeia documental. Segundo Svenonius (2000, p.3),

a efetividade de um sistema ao organizar a informação é em parte uma função de uma ideologia que expressa as ambições dos criadores e o que eles esperam arquivar.

Por exemplo, a aquisição de um documento está submetida, entre outras variáveis, aos objetivos correntes e prioridades da unidade e à natureza da unidade e de seus serviços. Há influência do perfil da unidade de informação também no processo de descrição do conteúdo de um documento. $\mathrm{O}$ assunto de um documento é definido para atender com mais eficácia aos interesses específicos dos seus usuários.

Uma das estratégias mais comuns adotadas pelas unidades de informação é a atuação em colaboração com instituições similares, visando à formação de uma rede para troca de documentos (em qualquer fase da cadeia documental, da aquisição à difusão) e informações de interesse comum. Estas redes colaborativas podem formar-se em torno de uma unidade central ou de modo 
descentralizado e ainda interligar instituições localizadas em uma mesma cidade ou em regiões diferentes.

\section{CONSIDERAÇÕES FINAIS}

Neste trabalho, procuramos articular conceitos tradicionais do campo da ciência da informação com outros próprios do ambiente digital propiciado pelas TICs. Em d'Andréa (2005), estes conceitos foram a base para a proposição de uma metodologia de análise de sites que procurasse identificar os processos e estratégias de produção e organização de informações adotadas em sites (no caso, sites sobre localidades turísticas). Para isso, propusemos quatro sub-roteiros de análise: Organização da Informação na WWW, Documentos e Hipertextualidade, Conteúdo específico (no caso, Informação Turística, não contemplada neste artigo) e Unidades de Informação.

O primeiro sub-roteiro foi elaborado a partir de técnicas e estratégias tradicionalmente utilizadas nas unidades de informação e relatadas por Guinchat e Menou (1994), assim as mudanças e novidades incorporadas em um contexto digital e apreendidas pelo conceito de arquitetura da informação detalhado por Rosenfeld e Morville (1998).

O sub-roteiro Documentos e Hipertextualidade baseiase na reformulação do conceito de documento proposta por Pédauque (2003), compreendido aqui como uma evolução do documento tradicional descrito por Guinchat e Menou (1994). O uso das possibilidades da hipertextualidade para construção do sentido do documento e de seu reconhecimento social foram os tópicos centrais analisados. Por fim, o quarto sub-roteiro busca identificar características das unidades de informação responsáveis pela concepção e/ou manutenção dos sites analisados, visando a compreender sua influência na produção/coleta e organização das informações do site.

Artigo submetido em 27/10/2005 e aceito em 13/03/2007.

\section{REFERÊNCIAS}

ALEXANDRE, Jan; TATE, Ann. Evatualing web resources. Widener University Homepage. 1996. Disponível em: < http://www2.widener.edu/ Wolfgram-Memorial-Library/webevaluation/webeval.htm >. Acesso em: 19 maio 2004.

BARRETO, Aldo. As tecnologias intensivas de informação e comunicação e o reposicionamento dos atores do setor. In: INFO 97, 1997, Cuba. Anais eletrônicos... Disponível em: <www.alternex.com.br/ حaldoibct/cuba.htm >. Acesso em: 2007.
- Mudança estrutural no fluxo do conhecimento: a comunicação eletrônica. Ciência da Informação, Brasília, v. 27, n. 2, p. 122-127, maio/ago. 1998.

BORKO, H. Information science: what is it?. American Documentation, Washington, v. 19, n. 1, p. 3-5, Jan. 1968

CARVAlHO, Natália Guiné de Mello. Agências de notícias na Internet como fonte de informação para negócios. 2001. 122 f. Dissertação (Mestrado em Ciência da Informação)- Escola de Ciência da Informação, UFMG, Belo Horizonte, 2001.

D'ANDRÉA, Carlos F. de B. Estratégias de organização e produção de informações na www: uma análise de sites turísticos. 2005. $192 \mathrm{f}$. Dissertação (Mestrado em Ciência da Informação) - Escola de Ciência da Informação, UFMG, Belo Horizonte, 2005.

GUINCHAT, Claire; MENOU, Michel. Introdução geral às ciências e técnicas da informação e da documentação. Brasília: MCT: CNPq: Ibict, 1994.

LE COADIC, YvesFrançois. A ciência da informação. Brasília: Briquet de Lemos, 1996.

MIRANDA, Antonio. Sociedade da informação: globalização, identidade cultural e conteúdos. Ciência da Informação, Brasília, v. 29, n. 2, p. 78-88, maio/ago. 2000.

NASCIMENTO, Niraldo José. Avaliação de sites sobre gestão do conhecimento na world wide web: um estudo exploratório. 2000. 139 f. Dissertação (Mestrado em Ciência da Informação) - Escola de Ciência da Informação, UFMG, Belo Horizonte, 2000.

PARENTE. André. O virtual e o hipertextual. Rio de Janeiro: Pazulin, 1999.

PÉDAUQUE, Roger T. Document: forme, signe et médium, les reformulations du numérique. Disponível em: <http:// archivesic.ccsd.cnrs.fr/documents/archives0/00/00/05/11/ sic_00000511_01/sic_00000511.pdf>. Acesso em: 20 out. 2004.

ROSENFELD, Louis; MORVILLE, Peter. Information architecture for the world wide web. Sebastopol: O'Reilly, 1998.

SARACEVIC, T. Ciência da informação: origem, evolução e relações. Perspectivas em Ciência da Informação, Belo Horizonte, v. 1, n. 1, p. 41 62, jan./jun. 1996.

SMITH, Alastair G. Testing the surf: criteria for evaluating Internet information resources. Public-Access Computer Systems Review, v. 8, n. 3, 1997. Disponível em: <http://info.lib.uh.edu/pr/v8/n3/ smit8n3.html>. Acesso em: 15 fev. 2004.

SVENONIUS, Elaine. The intellectual foundation of information organization. Cambridge, Mass.: MIT Press, 2000.

TILLMAN, Hope N. Evaluating quality on the net. 2003. Disponível em: <http://www.hopetillman.com/findqual.html>. Acesso: 02 ago. 2003.

TOMAÉL, M. et al. Avaliação de fontes de informação na Internet: critérios de qualidade. Informação e Sociedade, João Pessoa, v. 11, n. 2, p. 13-35, 2001.

TRISTÃO, Márcio. A arquitetura da informação segundo Lou e Peter. Webinsider. 2002. Disponível em: <http://webinsider.uol.com.br/ vernoticia.php/id/1397>. Acesso em: 20 out. 2004.

VILELLA, Renata Moutinho. Conteúdo, usabilidade e funcionalidade: três dimensões para a avaliação de portais estaduais de governo eletrônico na web. 2003. 263 f. Dissertação (Mestrado em Ciência da Informação)- Escola de Ciência da Informação, UFMG, Belo Horizonte, 2003. 